\title{
NIELSEN NUMBERS OF HOMOTOPICALLY PERIODIC MAPS ON INFRASOLVMANIFOLDS
}

\author{
CHRISTOPHER K. MCCORD
}

(Communicated by Thomas Goodwillie)

\begin{abstract}
A well-known lower bound for the number of fixed points of a selfmap $f: X \rightarrow X$ is the Nielsen number $N(f)$. Unfortunately, the Nielsen number is difficult to calculate. The Lefschetz number $L(f)$, on the other hand, is readily computable but usually does not estimate the number of fixed points. In this paper, we show that on infrasolvmanifolds (aspherical manifolds whose fundamental group has a normal solvable group of finite index), $N(f)=L(f)$ when $f$ is a homotopically periodic map.
\end{abstract}

\section{Estimating AND COMPUTING NIELSEN NUMBERS}

In the past decade, considerable interest in Nielsen theory has focused on the relation between Nielsen numbers and Lefschetz numbers. The Nielsen number provides a lower bound on the number of fixed points of a self-map. It is a homotopy invariant, and on all compact manifolds (except surfaces with negative Euler characteristic), every homotopy class contains a map $f$ with exactly $N(f)$ fixed points. Unfortunately, $N(f)$ is not readily computable from its definition, so other methods of computation must be sought.

One natural method to consider is the possibility of computing Nielsen numbers from Lefschetz numbers. Roughly speaking, the Nielsen number provides a geometric count of the fixed point set, while the Lefschetz number provides an algebraic condition. The starting point for relating the two is the theorem of Brooks, Brown, Pak, and Taylor [3], that $N(f)=|L(f)|$ for all self-maps on tori. This was extended by Anosov [1] to nilmanifolds (homogeneous spaces of nilpotent Lie groups). At least for the present, we know of no larger class of manifolds for which $N(f)=|L(f)|$ for all self-maps. The next natural classes of manifolds to consider, infranilmanifolds and solvmanifolds, provide counterexamples. Indeed, there are counterexamples on the Klein bottle, which is both a solvmanifold and an infranilmanifold.

However, there are still important connections between Lefschetz numbers and Nielsen numbers for manifolds other than nilmanifolds. In [8] the author showed that $N(f) \geq|L(f)|$ for all self-maps on solvmanifolds (homogeneous spaces of solvable Lie groups) and in [9] extended this inequality to all maps on

Received by the editors February 27, 1992 and, in revised form, April 27, 1992.

1991 Mathematics Subject Classification. Primary 55M20. 
infrasolvmanifolds (manifolds which have a finite regular cover by a solvmanifold). That is, on these manifolds, the Lefschetz number provides a lower bound (but not necessarily a sharp one) for the number of fixed points. Moreover, the results of [9] also imply that for self-maps on infranilmanifolds (manifolds that have a finite regular cover by a nilmanifold) there is a formula which computes the Nielsen number of a self-map in terms of Lefschetz coincidence numbers of lifts of the map.

Another line of development has been to impose extra conditions on the map, which allow these inequalities to be converted to equalities. Kwasik and Lee [6] showed that, if $f$ is a homotopically periodic self-map (i.e., some power of $f$ is homotopic to the identity), then $N(f)=L(f)$. This result was extended to homotopically periodic maps of solvmanifolds by Lee [7].

In this note, we extend this last line of results from infranilmanifolds and solvmanifolds to their natural common generalization: infrasolvmanifolds. Namely, we prove:

Theorem 1. If $M$ is a compact infrasolvmanifold and $f: M \rightarrow M$ is a homotopically periodic self-map on $M$, then $N(f)=L(f)$.

The proof proceeds by using the techniques of [9] to lift the problem from homotopically periodic self-maps on infrasolvmanifolds to homotopically periodic maps on solvmanifolds, and then applying Lee's result for solvmanifolds. To develop the ingredients needed for the proof, we review the necessary facts about infrasolvmanifolds in the next section, summarize the relevant parts of [9] in $\S 3$, and present the proof in the last section.

\section{INFRASOLVMANIFOLDS}

In this section, we briefly review the topology of infrasolvmanifolds. Infrasolvmanifolds can be defined in several ways. The constructive approach to infrasolvmanifolds begins with a solvable connected simply connected Lie group $S$. Consider the Lie group $G=S \rtimes K$, where $K$ is a compact subgroup of $\operatorname{Aut}(S) . G$ acts on $S$ by $(s, \alpha) \cdot s^{\prime}=s \alpha\left(s^{\prime}\right)$. If $\pi \subset G$ is a torsionfree subgroup with finite projection $\Phi$ onto $K$, then $M=\pi \backslash S$ is an infrasolvmanifold. $M$ is connected and is compact if and only if $\pi$ is uniform in $S \rtimes K$ or, equivalently, if and only if $\Gamma=\pi \cap S$ is uniform in $S$. We will restrict ourselves to the compact case. (Though presented differently, this formulation is equivalent to that found in $[2,5]$.)

If $\Phi$ is solvable, then $M$ is a homogeneous space of a solvable Lie group and is called a solvmanifold. Every infrasolvmanifold has a finite regular cover by a solvmanifold. In fact, this characterizes infrasolvmanifolds. Namely, if $M=\pi \backslash S$, let $\pi_{s}$ be the solvradical of $\pi$-the unique maximal normal solvable subgroup of $\pi$-and let $\Phi_{s}$ be the solvradical of $\Phi$. If $\rho: \pi \rightarrow \Phi$ is the projection homomorphism, $\pi_{s}=\rho^{-1}\left(\Phi_{s}\right)$. Let $\widetilde{M}=\pi_{s} \backslash S$. Then $\widetilde{M}$ is a solvmanifold covering $M$, with finite covering group $\pi / \pi_{s} \cong \Phi / \Phi_{s}$.

Note that the universal cover $S$ of $M$ is contractible, so $M$ is aspherical with $\pi_{1}(M) \cong \pi$. In fact, compact infrasolvmanifolds are determined up to homeomorphism by their fundamental group. We can classify precisely which groups can occur as the fundamental group of an infrasolvmanifold. Recall that for any property $\mathrm{P}$ of groups, a group $G$ is virtually $\mathrm{P}$ if there is a normal subgroup of finite index $H$ which has property $\mathrm{P}$. Similarly, a group 
is poly-P if there is a normal series $\left\{G_{i}\right\}$ for $G$ such that each subquotient $G_{i} / G_{i+1}$ has property P. In particular, $G$ is polycyclic if there is a normal series with $G_{i} / G_{i+1} \cong \mathbb{Z}$. There is a one-to-one correspondence between homeomorphism classes of infrasolvmanifolds and isomorphism classes of virtually polycyclic groups. These isomorphism classes can be equivalently described as isomorphism classes of poly-\{cyclic or finite $\}$ groups.

\section{NIELSEN NUMBERS AND COVERING SPACES}

While Nielsen numbers and Nielsen-type numbers can be defined in a wide variety of settings, it will suffice for our purposes to consider the setting of a compact manifold $M$ and a self-map $f: M \rightarrow M$. The fixed point set $\operatorname{Fix}(f)=\{x \in M \mid f(x)=x\}$ is then a compact subset, which we partition into fixed point classes by the following relation. If $x, y \in \operatorname{Fix}(f)$, then $x \sim y$ if there is a path $c$ in $M$ from $x$ to $y$ with $c \simeq f \circ c(\operatorname{rel}\{0,1\})$. Each fixed point class $F$ is then an isolated fixed point set, with a fixed point index $\operatorname{ind}(f, F)$ defined. A fixed point class is essential if its fixed point index $\operatorname{ind}(f, F)$ is nonzero, and the Nielsen number $N(f)$ is the number of essential fixed point classes. Let $\mathscr{E}(f)$ denote the set of essential fixed point classes of $f$. We can assume (after modifying $f$ by a homotopy, if necessary) that each essential class $F \in \mathscr{E}(f)$ consists of a single point and that all nonempty fixed point classes are essential.

We will need to understand how Nielsen numbers behave with respect to covering spaces. This question is studied in detail in $[9, \S 5]$. There, the more general phenomenon of coincidences between maps is considered; here, we specialize the results to the case of fixed points. Specifically, suppose $p: \widetilde{M} \rightarrow M$ is a finite regular cover of $M$ with fundamental group $\tilde{\pi}$ and covering group $\mathscr{D} \cong \pi / \tilde{\pi}$. A self-map $f: M \rightarrow M$ is covered by a map $\tilde{f}: \widetilde{M} \rightarrow \widetilde{M}$ if and only if $\operatorname{im}\left(f_{\sharp} \circ p_{\sharp}\right) \subseteq \operatorname{im}\left(p_{\sharp}\right)$ in $\pi$; that is, if and only if $\operatorname{im}\left(p_{\sharp}\right)$ is invariant under $f_{\sharp}$. The map $f$ is then also covered by $\beta \circ \tilde{f}$ for every $\beta \in \mathscr{D}$. If $x \in \operatorname{Fix}(f)$, then there is a unique $\beta \in \mathscr{D}$ such that $p^{-1}(x) \subset \operatorname{Fix}(\beta \tilde{f})$. If $\tilde{x} \in p^{-1}(x)$, then $\beta \tilde{f}$ around $\tilde{x}$ and $f$ around $x$ are conjugate via $p$, so $\operatorname{ind}(\beta \tilde{f}, \tilde{x})=\operatorname{ind}(f, x)$. Since $x$ is covered by $|\mathscr{D}|$ such points, we see that

$$
L(f)=\sum_{x \in \operatorname{Fix}(f)} \operatorname{ind}(f, x)=\frac{1}{|\mathscr{D}|} \sum_{\beta \in \mathscr{D}} \sum_{\tilde{x} \in \operatorname{Fix}(\beta \tilde{f})} \operatorname{ind}(\beta \tilde{f}, \tilde{x})=\frac{1}{|\mathscr{D}|} \sum_{\beta \in \mathscr{D}} L(\beta \tilde{f}) .
$$

Relating the Nielsen number $N(f)$ to the Nielsen numbers $N(\beta \tilde{f})$ of lifts requires more care. We must consider how the partitioning of $\bigcup_{\beta \in \mathscr{D}} \operatorname{Fix}(\beta \tilde{f})=$ $p^{-1}(\operatorname{Fix}(f))$ into fixed point classes relates to the partitioning of $\operatorname{Fix}(f)$ into fixed point classes. It is easy to see that $p$ maps fixed point classes in $\widetilde{M}$ to fixed point classes in $M$, so a fixed point class $\widetilde{F}$ of $\beta \tilde{f}$ lies entirely in some $p^{-1}(x)$. Since all points in $\widetilde{F}$ have the same index as $x, \widetilde{F}$ is essential if and only if $x$ is. It remains then to see how many distinct fixed point classes of $\beta \tilde{f}$ lie in $p^{-1}(x)$. If $F$ is a fixed point class of $f$ and $x \in F$, let $\tilde{\pi}_{F}$ denote the image of $\tilde{\pi}$ in $\pi_{1}(M, x)$ and let $\operatorname{Fix}\left(f_{\sharp}, F\right)=\left\{\omega \in \pi(M, x) \mid f_{\sharp}(\omega)=\omega\right\}$. In general, this quantity depends on the fixed point class $F$ but not on $x \in F$. Then $p^{-1}(x)$ is a union of $\left[\pi: \tilde{\pi}_{F} \cdot \operatorname{Fix}\left(f_{\sharp}, F\right)\right]$ fixed point classes, each containing $\left[\tilde{\pi}_{F} \cdot \operatorname{Fix}\left(f_{\sharp}, F\right): \tilde{\pi}_{F}\right]$ fixed points. 
As a way of combining all of this information, we introduce the Nielsen-type number

$$
\tilde{N}(f, \tilde{\pi})=\frac{1}{|\mathscr{D}|} \sum_{\beta \in \mathscr{D}} N(\beta \circ \tilde{f}) .
$$

$\tilde{N}(f, \tilde{\pi})$ does in general depend on $\tilde{\pi}$. The relation between $N(f)$ and $\tilde{N}(f, \tilde{\pi})$ can now be stated as follows:

Theorem 2 [9, Theorem 5.9]. If $\tilde{\pi}$ is a normal subgroup of $\pi=\pi_{1}(M)$ of finite index and $f: M \rightarrow M$ is a self-map with $f_{\sharp}(\tilde{\pi}) \subset \tilde{\pi}$, then $N(f) \geq \tilde{N}(f, \tilde{\pi})$, with equality if $\operatorname{Fix}\left(f_{\sharp}, F\right) \subset \tilde{\pi}_{F}$ for every $F \in \mathscr{E}(f)$.

\section{Proof of THE THEOREM}

If $\pi=\pi_{1}(M)$ and $\pi_{s}$ is the solvradical of $\pi, \pi_{s}$ is a characteristic subgroup of $\pi$. In particular, since $f$ is homotopically periodic, $f_{\sharp}: \pi \rightarrow \pi$ is an automorphism, and $f_{\sharp}\left(\pi_{s}\right)=\pi_{s}$. If $p: \widetilde{M} \rightarrow M$ is the finite regular covering of $M$ corresponding to $\pi_{s}$, then $f$ is covered by maps $\beta \circ \tilde{f}: \widetilde{M} \rightarrow \widetilde{M}$, where $\beta \in \mathscr{D}=\pi / \pi_{s}$. If $F \in \mathscr{E}(f)$ is an essential fixed point class of $f$ and $\beta \in \mathscr{D}$ is chosen so that $p^{-1}(F) \subset \operatorname{Fix}(\beta \tilde{f})$, then every fixed point class $\tilde{F}$ of $\beta \tilde{f}$ in $p^{-1}(F)$ has index

$$
\operatorname{ind}(\beta \tilde{f}, \tilde{F})=\left[\tilde{\pi}_{F} \cdot \operatorname{Fix}\left(f_{\sharp}, F\right): \tilde{\pi}_{F}\right] \operatorname{ind}(f, F) .
$$

Since $F$ is essential, $\operatorname{ind}(f, F) \neq 0$ and $\operatorname{ind}(\beta \tilde{f}, \tilde{F}) \neq 0$. Then $|\operatorname{ind}(\beta \tilde{f}, \tilde{F})|$ $=1\left[8\right.$, Theorem 1], so $\left[\tilde{\pi}_{F} \circ \operatorname{Fix}\left(f_{\sharp}, F\right): \tilde{\pi}_{F}\right]=|\operatorname{ind}(f, F)|=1$. In particular, $\tilde{\pi}_{F} \cdot \operatorname{Fix}\left(f_{\sharp}, F\right)=\tilde{\pi}_{F}$ and $\operatorname{Fix}\left(f_{\sharp}, F\right) \subset \tilde{\pi}_{F}$. Therefore,

$$
N(f)=\frac{1}{|\mathscr{D}|} \sum_{\beta \in \mathscr{D}} N(\beta \circ \tilde{f}) \text { and } L(f)=\frac{1}{|\mathscr{D}|} \sum_{\beta \in \mathscr{D}} L(\beta \circ \tilde{f}) .
$$

It suffices then to show that $N(\beta \circ \tilde{f})=L(\beta \circ \tilde{f})$ for every $\beta \in \mathscr{D}$. Since $f$ is homotopically periodic, some $f^{n} \simeq \mathrm{id}$, and each $(\beta \circ \tilde{f})^{n}$ is homotopic to a covering transformation. But $\mathscr{D}$ is finite, so for each $\beta$ there is an $m$ such that $(\beta \circ \tilde{f})^{n m} \simeq \mathrm{id}$. That is, each $\beta \circ \tilde{f}$ is homotopically periodic. Now, Lee's result for homotopically periodic maps on solvmanifolds [7, Theorem 2] applies, and $N(\beta \circ \tilde{f})=L(\beta \circ \tilde{f})$ for every $\beta$.

\section{REFERENCES}

1. D. V. Anosov, The Nielsen number of maps of nil-manifolds, Russian Math. Surveys $\mathbf{4 0}$ (1985), 149-150.

2. L. Auslander and F. E. A. Johnson, On a conjecture of C. T. C. Wall, J. London Math. Soc. (2) 14 (1976), 331-332.

3. R. Brooks, R. Brown, J. Pak, and D. Taylor, Nielsen numbers of maps of tori, Proc. Amer. Math. Soc. 52 (1975), 398-400.

4. E. Fadell and S. Husseini, On a theorem of Anosov on Nielsen numbers for nilmanifolds, Nonlinear Functional Analysis and its Applications (Maratea, 1985), NATO Adv. Sci. Inst. Ser. C: Math. Phys. Sci., vol. 173, Reidel, Dordrecht and Boston, MA, 1986, pp. 47-53.

5. F. T. Farrell and L. E. Jones, Classical aspherical manifolds, CBMS Lecture Notes, vol. 75, Amer. Math. Soc., Providence, RI, 1990. 
6. S. Kwasik and K. B. Lee, The Nielsen numbers of homotopically periodic maps of infranilmanifolds, J. London Math. Soc. (2) 38 (1988), 544-554.

7. K. B. Lee, Nielsen numbers of periodic maps on solvmanifolds, Proc. Amer. Math. Soc. 116 (1992), 575-579.

8. C. McCord, Nielsen numbers and Lefschetz numbers on solvmanifolds, Pacific J. Math. 147 (1991), 153-164.

9. C. McCord, Estimating Nielsen numbers on infrasolvmanifolds, Pacific J. Math. 154 (1992), 345-368.

Institute for Dynamics, Department of Mathematics, University of Cincinnati, Cincinnati, OHIO 45221-0025

E-mail address: chris.mccordQuc.edu 